\title{
Review
}

\section{Aggression in Huntington's Disease: A Systematic Review of Rates of Aggression and Treatment Methods}

\author{
Caroline A. Fisher ${ }^{\mathrm{a}, \mathrm{b}, *}$, Katherine Sewell ${ }^{\mathrm{b}}$, Anahita Brown ${ }^{\mathrm{b}}$ and Andrew Churchyard ${ }^{\mathrm{c}, \mathrm{d}}$ \\ ${ }^{a}$ Child and Youth Mental Health Service, Adolescent Inpatient Psychiatric Unit, Box Hill Hospital, Eastern Health, \\ Melbourne, Australia \\ ${ }^{\mathrm{b}}$ Brain Disorders Program, Royal Talbot Rehabilitation Centre, Austin Health, Melbourne, Australia \\ ${ }^{\mathrm{c}}$ Huntington's Disease Service, Calvary Health Care Bethlehem, Melbourne, Australia \\ ${ }^{\mathrm{d}}$ School of Psychological Sciences, Faculty of Medicine, Nursing and Health Sciences, Monash University, \\ Melbourne, Australia
}

\begin{abstract}
Aggression is commonly reported in individuals with Huntington's disease (HD). While correlating factors for aggression are often speculated about, features that are associated with, and contribute to, aggression in this population have not been clearly determined. This systematic review investigates rates of aggression and treatment options for aggression in HD. A number of key findings were revealed. Studies reporting on rates of aggression revealed that its prevalence is high, falling between 22 and 66 percent in the majority of studies. Aggression may be more common in males with HD, and is also found in higher rates in individuals who experience frequent falls, have obsessive-compulsive symptoms and suicidal ideation. There is little research investigating antecedents for aggression in HD. A wide variety of psychotropic medications have been reported in the literature to treat individuals with HD and aggressive behaviour. However, due to methodological limitations, no treatment recommendations can be made, based on the current literature. Two non-medication therapies have been investigated, behaviour support and sensory modulation intervention. However, again, due to methodological limitations with these studies, further research is needed before they can be recommended as frontline interventions. This review highlights the need for further methodologically rigorous studies investigating the treatment of aggression in HD.
\end{abstract}

Keywords: Aggression, Huntington's disease, prevalence, treatment, therapy

\section{INTRODUCTION}

Huntington's disease (HD) is a neurodegenerative genetic disorder with autosomal dominant inheritance $[1,2]$ that is characterised clinically by motor abnormalities (chorea, falls) [3], cognitive impairment [4, 5]

${ }^{*}$ Correspondence to: Dr. Caroline Fisher, Child and Youth Mental Health Service, Adolescent Inpatient Psychiatric Unit, Box Hill Hospital, Eastern Health, 5 Arnold Street, Box Hill, Victoria 3128, Australia. Tel.: +61 39092 6725; Fax: +61 39348 2766; E-mail: Caroline.Fisher@easternhealth.org.au. and psychiatric disturbance [6]. High rates of aggression have also been reported. Aggression is one of the primary causes of hospitalisation in this population [7], is associated with higher rates of nursing home placement [8] and places family members, carers and other clients at risk of assault. While a number of previous texts have speculated on factors that may contribute to aggressive behaviour in HD [9-11] correlating clinical symptoms and antecedents for aggression remain unclear. There are also presently no recommended clinical guidelines on how to manage aggression effectively in Huntington's disease. 
This paper has two aims. The first is to review the published evidence on rates of aggression in Huntington's disease and identify correlating factors as well as behavioural, situational or environmental antecedents for this behaviour. The second is to review the published evidence on treatment strategies for aggression in $\mathrm{HD}$, including both pharmacological and nonpharmacological interventions. It is anticipated that these investigations will provide a clearer understanding of aggression in Huntington's disease and help to inform future research into this distressing behavioural sequelae.

\section{Identification and classification of studies}

Aggression is an externalised behaviour that can be directly observed and measured via observation from carers, family members and hospital staff. In their seminal text Bushmann and Anderson define aggression as a noxious stimuli that is delivered with the intent to harm, threaten or reject the recipient [12]. For the purposes of this paper, aggression is defined as: any behaviour that attempts to inflict uninvited force, harm or damage to a person or inanimate object, or verbal behaviour that is delivered in a intimidating manner (swearing, yelling, shouting, insults or threats). Irritability has also been investigated in a number of studies of HD sufferers [e.g. 13, 14] and is sometimes grouped together in symptoms clusters with aggression [15]. Irritability, however, is generally conceptualised as an internalised mood state [16] that requires client participation to rate $[17,18]$, and is not necessarily possible to monitor via observations. As such, it is difficult to accurately gauge irritability across all stages of HD as persons with advanced HD may not be able to provide ratings of their internal mood state and irritability levels, due to cognitive and communication impairments. Due to the heterogeneity between these clinical variables the review has focused specifically on aggression as an overt, observable behaviour.

To identify original studies reporting on aggression in Huntington's disease a database search of PubMed, Medline and PsycInfo to September 20th 2014 was performed. The search term "Huntington's" was combined with the terms "aggression", "assault" and "violence". This revealed a total of 52 studies, after duplicates were removed. A further 13 studies were identified following reference section searches of the papers initially obtained, as well as a three further studies known to the authors. These 68 papers were then examined and studies were included if they meet at least one of the two inclusion criteria: I) original research studies examining rates of aggression, correlates with aggression or antecedents for aggression in individuals with Huntington's disease, II) original research studies outlining treatment approaches for aggression in Huntington's disease. Fourteen papers meet inclusion criteria I. Nineteen studies meet inclusion criteria II. Studies were excluded if they: involved animal rather than human subjects, if the HD participants were included in a group study with other disorders and the HD data could not be separated out, if the data on aggression could not be separated out from other sequelae (e.g. irritability), if they were review papers not containing original research, if they did not contain any subjects with Huntington's disease, and if they were not investigating aggression or the treatment of aggression.

\section{Rates of aggression in $H D$}

\section{Characteristics of studies}

Of the studies that meet inclusion criteria I, fourteen papers from thirteen individual studies are summarised in Table 1 [7, 19-31]. The results of one study were spread across two papers, and thus were combined so that the reporting was not duplicated [7, 21]. A further study [32], which reported on aggression only as a correlate of suicidal ideation, rather than as an individual variable of interest, was not included in the table, but is reported in the Associations/Correlations with Aggression section, below. For eleven of the studies the sample sizes ranged between 27 and 250 participants. Of these studies the most common type were case records reviews, followed by comparison studies with another group, (i.e. Alzheimer's disease [23] or a control group [26]), monitoring studies [25, 28], a behaviour scale development study [27] and a longitudinal symptoms monitoring study [30]. There were also a further two studies of data reviews from Huntington's registries containing very large samples [29, 31].

Gender ratios varied across the studies. Five studies $[22,24-26,30]$ contained a high percentage of female participants (60.4\% or higher), while the remaining studies had ratios ranging between 60:40 (in either direction) and even 50:50 distribution. The average age of the participants for the studies in which this figure was reported (or was calculable via a mean weighted average), ranged between 45.6 years and 57.2 years. Four studies contained at least one participant with juvenile onset Huntington's (JHD) [19, 20, 23, 27] and in one study JHD participants were excluded [26]. 


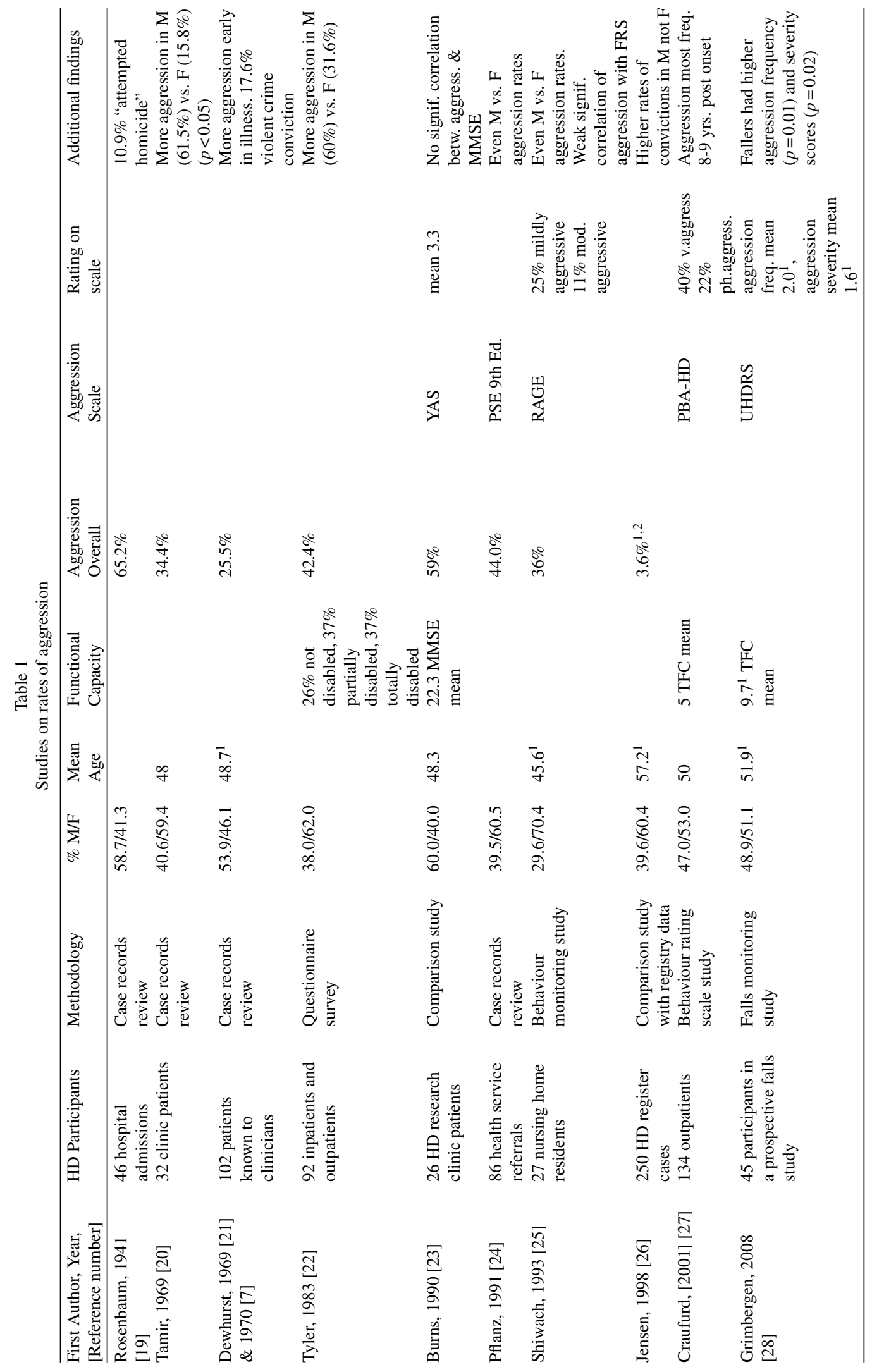




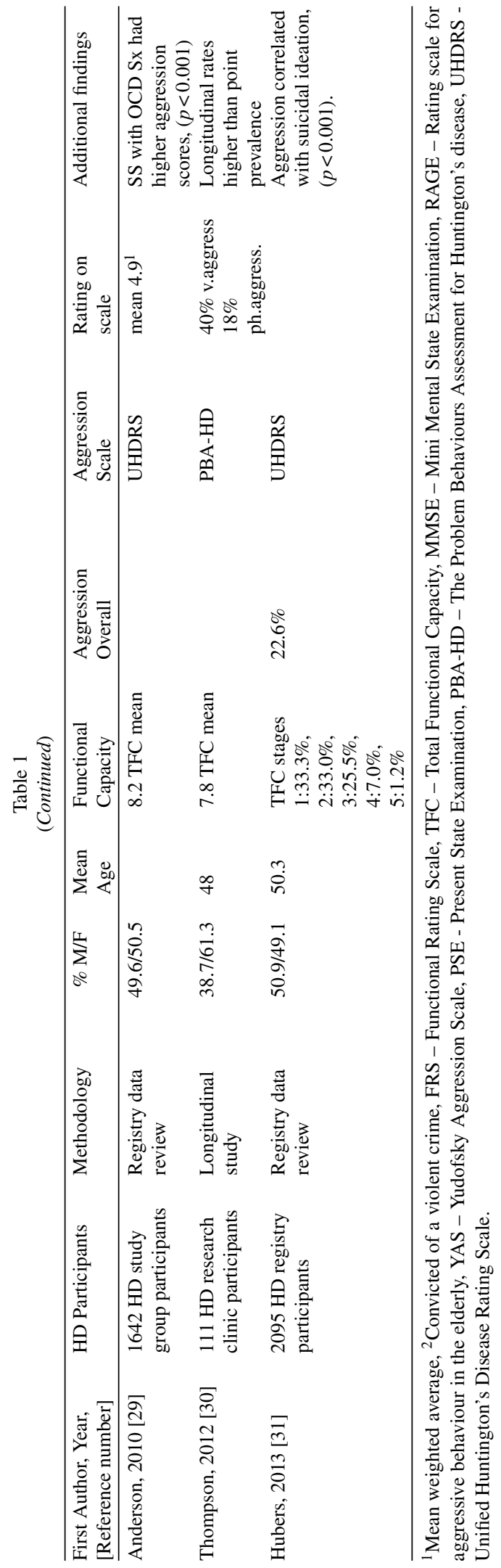


The diagnosis of HD for participants, where reported, was based on clinical categorisation in the majority of studies [19, 20, 23, 24, 29], or inclusion on an HD registry [26]. Three studies contained confirmed HD gene mutation carriers $[27,30,31]$. In one of these studies [31] 98 percent were motor symptomatic (thus, up to two percent of this sample may have had premanifest HD). Average CAG repeat mutations in these three studies ranged between 44 and 46 (calculated via the mean weighted average for Hubers, et al.). As a number of the studies were case records reviews (incorporating the patient's behaviour over a number of years) mean disease duration was often not reported. However, where this was available, average disease durations were 5.5 years [30], 5.7 years [31], 7.0 years [28, 29], 9 years [27] and an estimate of 10 years [22]. A number of differing measures were used to calculate the participants' functional capacity, including Mini Mental State Examination [33] and Total Functioning Capacity (TFC) [34] means, TFC stages and study developed classifications [e.g. 22].

\section{Rates of aggressive behaviour}

Rates of aggression (any type) overall were high in the majority of studies, ranging between 22.6 percent and 65.2 percent, across eight studies [7, 19-25, 31]. The highest rate was reported in a case records review study, that analysed patient file information, potentially spanning many years for each client [19), followed by a group comparison study that analysed the responses of family/caregivers on a questionnaire about both current and past behaviour [23]. The lowest rate was found in a large registry data review study utilising scores on the behavioural subscale of the Unified Huntington's Disease Rating Scale (UHDRS) [31]. A low rate was also found in a case records review study reporting the primary reason for admission to hospital in the HD cohort $[7,21]$. This data may represent an underestimation of aggression, in total, in this cohort as others in the study may also have exhibited aggressive behaviour that did not result in a hospital admission. In one further study [26] that only reported on convicted crimes, rates of conviction for violent crimes were 3.6 percent. This result differs significantly from reported rates of overall aggression in the other studies. It may indicate that, at least in recent years, aggression perpetrated by HD sufferers is either not commonly reported to the police, or infrequently prosecuted.

\section{Characteristics of the aggressive behaviour}

A number of studies did not utilise a recognised scale, or scoring system to rate or evaluate aggres- sion [7, 19-22], making it difficult to draw any further conclusions about the nature, severity or frequency of aggression in these studies. When a rating scale was used, the UHDRS aggression items were the most common [28, 29, 31, 32]. The Problem Behaviours Assessment for Huntington's Disease (PBA-HD) [27, 30], Rating Scale for Aggressive Behaviour in the Elderly (RAGE) [25], Yudofsky Aggression Scale [23] and Present State Examination, 9th Edition [24], were also utilised. Despite the comprehensive nature of some of these scales in assessing rates, types and severity of aggression, the full findings were not always reported, with overall subscale means for aggression the most common type of data provided.

The UHDRS Behaviour Assessment [34] has a Disruptive and Aggressive Behaviour subscale, with descriptors that all relate to aggression. These include: threatening behaviour, physical violence, verbal outbursts, and threatening, foul or abusive language. The UHDRS rates these behaviours according to both severity and frequency, but does not provide information separately on the different types of aggression (physical, verbal and towards furniture/property). Three papers utilised this scale. In a falls monitoring study with 45 HD participants [28] the mean weighted average for severity was 1.6 (rates between slight, questionable and mild), and the mean frequency was 2.0 (consistent with a classification of sometimes). In a large sample registry data review [29] the aggression score was calculated by multiplying the frequency score by the severity score, providing a mean weighted average of 4.9. Little further information can, thus, be drawn about the severity, frequency or nature of the aggression in this sample. In a second large sample registry data review [31], the authors indicated that aggression was also calculated by multiplying the frequency total by the severity total to produce an overall rating. This score does not appear to have been reported, however. Instead aggression was reported as the number of participants who exhibited aggression in any form, at any level of severity (i.e. cases with aggression $\geq 1$ ), at a rate of $22.6 \%$.

Two studies employed the Problem Behaviours Assessment for Huntington's Disease (PBA-HD) $[27,30]$ to rate aggressive behaviour. This is a semistructured behavioural interview, which rates both the frequency and severity of a range of problem behaviours. Information is provided from both patients and caregivers about behaviour over the preceding four weeks. In the original development of the scale [27] the items with a clear relationship to aggression are Temper, verbal outbursts (appearing in subsequent studies 
as Verbal outbursts) and Threatening behaviour, violence (appearing in subsequent studies as Physical aggression). The first study reported rates of verbal outbursts as 40 percent and physical aggression as 22 percent [27]. In the second study [30], exact numerical figures were not provided, but careful inspection of the provided bar graph figure revealed point prevalence rates that appear to correspond with 40 percent for verbal outbursts and 18 percent for physical aggression. Longitudinal ratings (reports of the behaviour at any time point during the study) appear to correspond to 75 percent for verbal outbursts and 49 percent for physical aggression. The actual duration of the longitudinal follow-up in this study is unclear, but appears to be around 6.5 years (mean of five assessments per participant, mean time between assessments 1.3 years). More specific information about aggression frequency and severity ratings are not provided in either study, despite these variables being collected by the PBA-HD.

The RAGE [35] was another measure of aggression employed in one of the studies [25]. Authors indicated that this scale rates behaviour over a three-day observation period. It consists of 23 items. Nineteen of these gauge observable behaviour, with some of these items directly classifiable as overt aggressive behaviour (e.g. shouted, yelled or screamed; attempted to bite, scratch, pinch or hit others, etc.). Three items gauge consequences of the aggressive behaviour (termed as the non-behavioural items) and one item determines a global judgement for overall aggressiveness. The authors reported the data for 17 of the items on this scale, by frequency (occurred once in three days, occurred everyday in the past three days, occurred more than once everyday in the past three days) in the 27 participants. By far the most common form of aggressive behaviour was found to be: shouted, yelled or screamed (33\% with positive ratings), with the majority (29.6\%) exhibiting this just once in three days and a smaller number $(3.7 \%$, i.e. 1 out of 27 participants) exhibiting it more than once a day, over the three days. Attempting to bite, scratch, pinch or hit others, was the most common form of physical aggression (11.1\% exhibited this at least once, with $3.7 \%$ exhibiting this more than once a day). Destroying property or throwing things around angrily occurred in $7.4 \%$ of participants, at a frequency of once in three days for all. The number of participants attempting to kick $(3.7 \%)$ or hit others $(3.7 \%)$ was low, but when displayed, this behaviour was frequent (more than once a day for both). Importantly however, not all the items from the RAGE were included in the results table. The results for "Pushed or shoved others" were not included in the results table, although this behaviour was reported as occurring in one in five participants, in the text (pg. 45). Overall, $25 \%$ of participants were rated as mildly aggressive, and $11.1 \%$ as moderately aggressive.

One group comparison study utilised the Yudofsky Aggression Scale. Information provided by the authors [23] indicated that it is comprised of four components: verbal aggression against self, physical aggression against self, aggression against objects and aggression against other people. During the data collection additional questions were also asked of relatives about: the most recent outburst, what outbursts were usually like, the frequency of the outbursts and any precipitating factors. Despite this, only information about the mean score on the scale, the number of participants above a "cut-off point" for the presence of aggression (any type) and the percentage of participants with aggressive periods lasting longer than 24 hours (31\%), were reported. No information was provided about the types of aggression (despite data being collected for this within the scale). Information on precipitating factors was also not analysed empirically, with only a general statement provided, which indicated "Aggressive outbursts were usually prompted by some event... Typical precipitants in the HD group included arguments with the spouse over money" (pg. 23). This was the only study in which reference was made to the collection of empirical data on precipitating, antecedent or triggering events for the aggressive outbursts.

Finally, the Present State Examination, 9th Edition [36] was also used to rate aggression in one study [24]. The measure is a semi-structured interview that rates participants on 140 items, which can then be used for diagnostic classification. It is unclear how many items relate to the classification of aggression. However, as presented in this study, the rating for aggression was considered a unitary item, with no further information provided other than prevalence rates, by gender $(45 \%$ in males and $44 \%$ in females).

\section{Associations/correlations with aggression}

A number of studies reported aggression results by gender. In three studies [24, 25, 27] relatively similar rates of aggression were reported across males and females. However, in a further three studies higher rates of aggression were observed in males [20, 22, 26]. In one study [7, 21] aggression was found to be more prevalent in the early, rather than later stages of the illness $(57.8 \%$ prevalence in the early phases; $9.8 \%$ in the middle phases and $2.0 \%$ in the terminal phase). In a second study [27] verbal aggression was found to be most 
prevalent in clients with 8-9 years illness duration, with physical aggression also showing a small increase at this illness stage compared to a relatively even rate of prevalence for physical aggression at earlier and later illness stages. Correlation analysis between aggression and cognitive or functional status was conducted in two studies [23, 25]. In one, no correlation was found between Mini Mental State Examination (MMSE) scores and aggression [23]. In the other [25], a significant but weak correlation was found between aggression and Functional Rating Scale scores (Pearson's correlation coefficient of $0.45, p<0.01$ ). This study does not explicitly state the direction of the correlation, however, from the information contained in the text it appears that poorer functional ratings were correlated with higher rates of aggression. In regard to physical variables, one study found significantly higher rates of aggression frequency and severity in participants with a high rate of falls, versus those without [28]. Psychiatric correlates of aggression were examined in three studies, all of which used the UHDRS to rate aggression. One found that participants with Obsessive Compulsive Disorder (OCD) symptoms had higher aggression scores [29], while two others found that rates of aggression were correlated with suicidal ideation $[31,32]$.

Data was also provided about rates of criminal behaviour that related to aggression in three studies. In one study [19] it was reported that 10.9 percent of participants had "attempted homicide" (pg.95), indicating a relatively high proportion of participants perpetrating physical aggression against others with force of potentially lethal intensity. It was further stated in the text that "In none was the homicidal attempt felt to be due to paranoid ideas - rather was it (sic) attributed to explosive irritability" (pg. 95). One study specifically examined the rates of criminality in HD sufferers, first degree relatives and the general population, by comparing HD and criminal registry data [26]. It found that overall crime rates were significantly increased in HD sufferers compared to first-degree relatives, but only for males. The increased rate of violent offences observed in male HD sufferers compared to both firstdegree relatives, and a matched control group, failed to reach statistical significance (HD 6\%; first degree relatives $1.5 \%$; matched controls $1 \%$ ). This contrasts somewhat with an earlier study [7] that reported high rates of conviction for a range of offenses in their HD sample (no control group), including assault (13\%), offenses against property $(12.7 \%)$ cruelty to children $(8.8 \%)$, malicious damage (1.9\%), blackmail (1\%) and arson $(1 \%)$.
Summary

Of the 14 studies that met the criteria for inclusion in this section of the review the majority were not specifically focused on examining aggression in HD. Rather, rates of aggression were collected along with a wide number of clinical variables, or measures of aggression were obtained as part of general Huntington's or psychiatric symptom scales and then analysed in relation to the main variable of interest. The majority of studies utilised clinical characterisation for the diagnosis of HD, with only three including confirmed gene positive cases, leaving the possibility open that a proportion of included participants may not have actually been afflicted with HD. The study methodologies ranged widely and the reporting of data from the clinical variables was poor in a number of studies. Overall, only 64 percent of the studies utilised a recognised $\mathrm{HD}$, behavioural or psychiatric scale for evaluating aggression.

Even with consideration given to these methodological issues, the results do appear to indicate that rates of aggression in HD are high, ranging between 22.6 percent and 65.2 percent in the majority of studies. Little information is available regarding the nature of the aggressive behaviour (e.g. verbal aggression, physical aggression, aggression to furniture/property). In the three studies that did report on types of aggression $[25,27,30]$ both verbal and physical aggression were found to occur, with verbal aggression having a higher prevalence. Aggression to property was reported to have been exhibited by just two participants in one study [25]. Overall, no studies reported empirical data on precipitating, antecedent or triggering events for the aggressive behaviour. One study that did collect this data (but did not report it empirically), indicated that aggressive outbursts were often triggered by arguments with spouses over money.

In the studies where gender differences were observed aggression was more prevalent in males. However, several studies reported even rates of aggression for both genders. Data on whether aggression becomes more or less prevalent in HD sufferers as the disease progresses is unclear. One study reported considerably higher rates of aggression in the early phases of the disease [7,21], a second indicated higher rates in the middle phase (8-9 years) [27], while in another aggression was weakly, but significantly, correlated with poorer ratings on a functional scale. Psychiatrically, there is evidence that aggression is more prevalent in clients with co-morbid OCD symptoms and also correlates with higher rates of suicidal ideation. Physically, it may also be more prevalent in 
sufferers that are also frequent fallers. In contrast to previous speculation $[10,11]$ there is presently little evidence that aggression in HD is associated with cognitive deterioration, irritability, personality factors, depression, psychosis or mania. Such associations may exist, but have yet to be thoroughly examined in the literature. Future research that focuses on investigating these factors carefully, using systematic evaluation of psychiatric symptoms and thorough cognitive and functional evaluation would be useful, as the methods employed thus far (e.g. MMSE, disease stage, FRS) may lack sufficient sensitivity to detect important associations. Finally, despite the high rates of aggression reported in most samples, the reported conviction rates for violent offenses in HD in the one recent study that examined this [26] were relatively low. This may reflect lower levels of reporting such episodes to police and/or infrequent prosecution of HD individuals.

\section{Treatment of aggression}

\section{Characteristics of studies}

Nineteen studies met inclusion criteria II, and are summarised in Table 2 [37-55]. All of the included studies had small samples sizes (1 to 6 participants), with eleven case studies $[39,41,42,44,46,48,49$, 51-54], seven case series [38, 40, 43, 45, 47, 50, 55] and one very small group study [37]. The majority of the studies were medication trials, with just two studies reporting non-medication interventions. Blass et al. [48] included a structured behaviour support plan, in addition to a poly medication trial, while Brown and Fisher [55] utilised sensory modulation intervention. Fifteen of the participants across the studies were female and twenty were male. The majority of participants were aged in their $30 \mathrm{~s}$ to $50 \mathrm{~s}$, with three younger participants, aged 16, 19 and 22 years, and three older participants, aged 60, 67 and 74 years, respectively. Where reported, the duration of HD from the onset of symptoms was variable. Participants who had been symptomatic for $10+$ years were reported in five studies $[38,43,47,50,55]$ while the remaining timelines reported were between 1 and 9 years. The functional or cognitive capacity of participants was reported in seven studies. In two studies intellectual capacity was reported, with IQ scores at the time of the intervention of 65 [51] and 89 [41]. Reported MMSE scores varied considerably, from participants who were unable to complete any MMSE tasks, scoring 0/30 [45, 48], moderately impaired participants, scoring 20/30 [54], to those who exhibited no difficulties on this measure, scoring 30/30 [42]. One study utilised the Shoulson's
Huntington's disease rating scale [46], with the participant in this study being classified as Stage V.

\section{Characteristics of the aggressive behaviour}

Only one study used a recognised behaviour scale, the Cohen-Mansfield Agitation Inventory (CMAI), to quantify the participant's behaviour [53]. For all remaining studies behaviour was categorised via the authors' descriptions. Nevertheless, there was sufficient information provided in most studies, to allow for the classification of the aggressive behaviour into three major types (physical, verbal or against furniture/objects), see Table 2. Physical aggression was the most common type, with a high prevalence of verbal aggression also reported. In only one study was aggression against property/furniture clearly described. The majority of studies made no mention of antecedents to the aggressive behaviour, with just three studies providing specific information about this. One study [55] outlined precursors for aggression individually for each of the participants. This study reported that for the female participant pacing, voicing hunger, verbal aggression and intrusiveness, where signs that often lead to behavioural escalation, whilst for the male participant loud noise from the television or co-residents was a precursor to verbal and physical aggression. The second study [42] stated that the participant self reported that her aggression was triggered by internal feelings of anger and irritability, which developed rapidly following minor irritants or changes in her routine. In the third study [48] the authors reported that the participant's behaviour was initially believed to be related to psychotic sequelae and delirium. However, following the implementation of a systematic behaviour evaluation period, it was then identified that the participant's behaviour escalated when he was asked to take medication or to interrupt a pleasant activity, such as walking or watching television. Problematic touching of female staff members was also found to occur during bathing or dressing activities.

\section{Treatment efficacy}

The quantification, measurement and evaluation of the effect of the therapeutic agents on aggression were generally poor. In just one study was an empirical measure of baseline behaviour provided [53] via a singular pre-treatment rating on the CMAI of 78. This study was also the only study to provide empirical outcome measures, via two further CMAI ratings during the medication treatment period with zuclopenthixole and medroxyprogresterone (CMAI of 56 at six weeks, and 57 at ten weeks). However, the statistical significance 


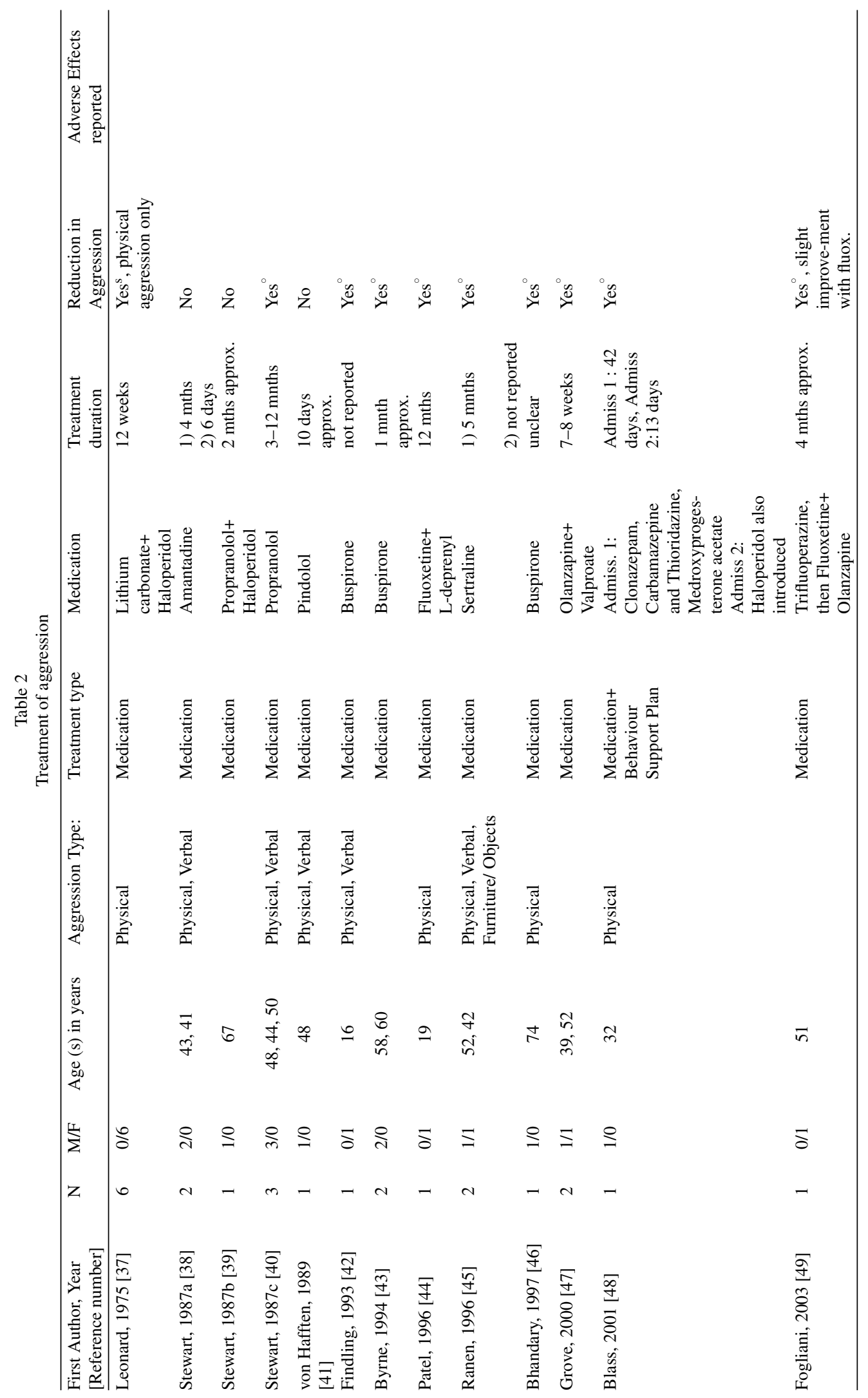




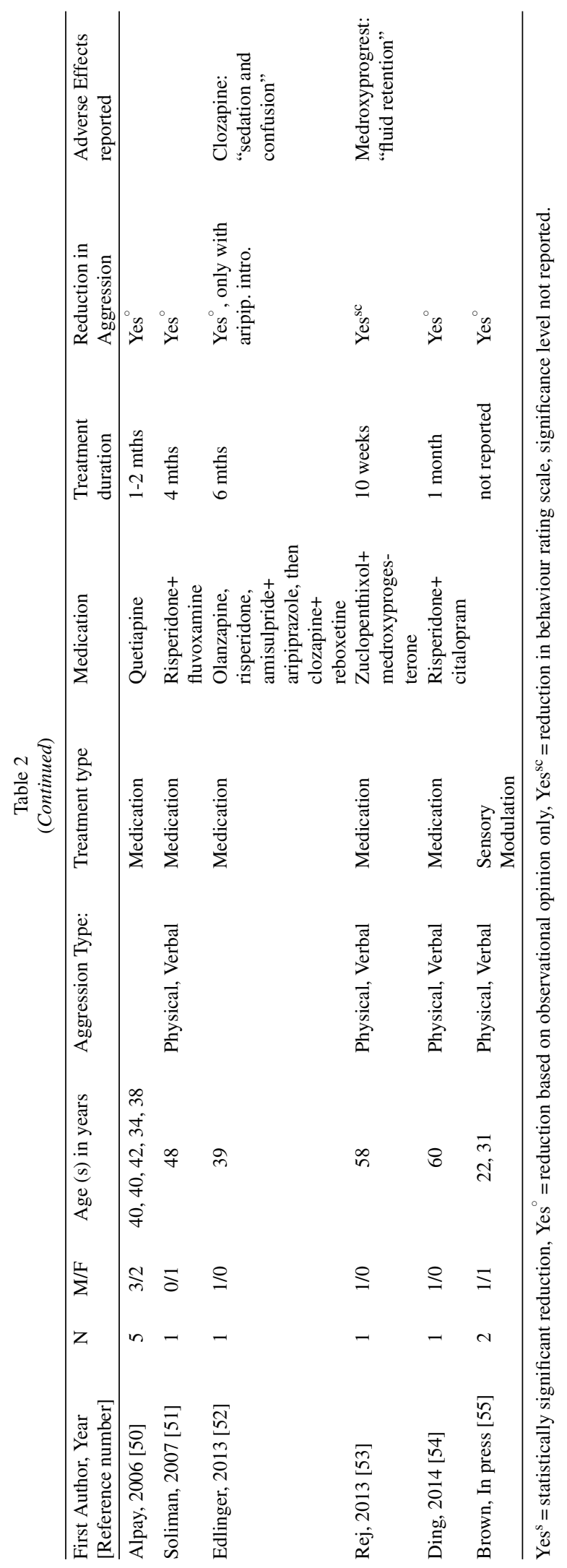


of the rate of change on this measure was not provided. Multiple baseline data for aggression were not reported in any studies. In one study [37] a placebo control condition was used, as well as blinded nursing staff who rated aggressive behaviour. However, the study reports that ratings were made only once at the end of each three week medication period, with no information provided about whether episodes of aggressive behaviour were recorded during the period (leaving the ratings open to potential confounds of the raters' memories over the entire three week period). This study reported a statistically significant positive effect of a combined pharmacotherapy approach [lithium carbonate and haloperidol) over placebo. This was the only study in which statistical analysis was applied to the behavioural data to determine the efficacy of the intervention. For all remaining studies the information provided about the efficacy of the treatment was based on the observational opinion of the authors.

Within the context of these methodological issues the majority of the studies reported positive effects of the therapeutic agents at reducing aggression (see Table 2). Antipsychotic medications were the most commonly reported pharmacotherapies. Haloperidol, a butyrophenone neuroleptic, was utilised with eight subjects, across three studies [37, 39, 48]. In one study it was reported to be effective at reducing aggression in six participants, when combined with lithium carbonate. However, in a single subject case study [39] no efficacious effect was reported on aggressive symptoms when haloperidol was combined with propranolol, a nonselective beta-blocker. Haloperidol was also introduced to a poly medication regime during a second admission in a further case study [48]. Quetiapine, an atypical antipsychotic, was also utilised in one study [50] containing five HD participants and was reported to have been affective at reducing aggression. Olanzapine, another atypical antipsychotic, was utilised in three participants across three studies [47, $49,52]$. In all of these studies it was used as part of a poly medication regime. It was reported as efficacious when combined with valproate [48], an anticonvulsant also with antipsychotic properties, and with fluoxetine [49], an antidepressant, although the authors in the later study attributed the small improvement in aggression primarily to the fluoxetine. Finally, olanzapine was also used as one of five psychotropic medications in another case study [52]. However, in this study the authors primarily attributed the improvement in aggression to the introduction of aripiprazole, another of the three antipsychotic agents employed in this case study. Thioridazine, a peperidine typical antipsychotic (now withdrawn in many countries due to cardiotoxicity and retinopathy at high does) was used in a poly medication regime in combination with behaviour support modifications [48], making its effect on aggression difficult to determine. Trifluoperazine, a phenothiazine neuroleptic, had little effect on aggression in one single case study [49], whilst zuclopenthixole, a thioxanthene neuroleptic, was reported to be efficacious in combination with, medroxyprogesterone, a progestogen, in another single case study [53]. However, the medroxyprogesterone is reported to have resulted in fluid retention and abdominal bloating. Side effects from the antipsychotic agent clozapine, were also reported in one study [52] and were described as sedation and confusion.

Two reported case studies combined the antipsychotic agent risperidone with selective serotonergic reuptake inhibitor antidepressants (SSRIs), specifically fluvoxamine [51] and citalopram [54]. In both of these studies aggression was reported to have decreased, following the introduction of the duo medication regimes. SSRIs were also used in several other studies. Sertraline was reported to improve aggressive symptoms in two HD clients in one case series study [45]. Fluoxetine was reported as the medication primarily responsible for reduction in aggression in one poly medication single case study [49], and was also reported to be effective when combined with Ldeprenyl in another [44]. The anxiolytic buspirone has also been utilised in four participants, across three studies with improvements reported in aggression in all cases [42, 43, 46].

Beta-adrenergic blocking agents were mixed in their effectiveness on aggressive symptoms in HD. Pindolol was reported to be ineffective in one single case study [41], as was propranolol, when combined with haloperidol, in another [39]. However, in a second study investigating the effect of propranolol alone, improvement in aggression was reported in three HD clients [40]. Finally, two medications generally employed for the treatment of movement disorders were also utilised. Amantadine alone did not improve aggressive behaviour in two participants in one study [38], whilst L-deprenyl when combined with fluoxetine (as indicated above) was reported to be effective in another [44].

In two studies, non-medication treatments were investigated. In one of these a behaviour support plan was instigated in conjunction with a poly medication trial over two separate inpatient admissions in a single case study [48]. This involved a systematic behaviour monitoring period, from which antecedents 
and precursors to problem behaviours were identified. A clear behaviour support plan was then developed involving a highly structured daily timetable routine, the minimisation of behaviour triggers where possible, positive verbal reinforcement of desired behaviour and rewards. The behaviour support plan was introduced two weeks after the new medication regime was commenced, and was reported to have resulted in dramatically improved behaviour within five days (in conjunction with the continued pharmacotherapy). The behaviour plan was reportedly successfully transferred to the patients' nursing home environment after discharge. It was also reinstituted on the inpatient unit when the patient was readmitted four months later, after a second period of suspected medication induced delirium.

In another study sensory modulation intervention was administered in a two case series [55]. This study reported on individual triggers for aggressive behaviour in both of the patients, as well as their individual sensory profiles. Sensory modulation treatment plans were developed for each client, utilising various sensory equipment (e.g. weighted blankets, puff massagers, click-clack balls), which were provided to clients to prevent the escalation of problem behaviour. The duration of the intervention was not specified, but was also reported to have resulted in a reduction in aggressive behaviour in both clients.

\section{Summary}

A wide variety of psychotropic medications have been reported in the literature to treat individuals with $\mathrm{HD}$ and aggressive behaviour. However, the published studies have very small sample sizes and poor measurement and evaluation of the effect of the therapeutic agents on aggression. No randomised controlled trials were revealed by the search, and none of the case studies or case series reports employed multiple baseline measures of aggression by frequency, type or severity. Two studies attempted to empirically quantify the effect of the medication on behaviour, however, each of these studies still contained methodological problems. Only two studies reported non-pharmacological measures to treat aggression in HD. Sensory modulation intervention was utilised in one study, whist behaviour support intervention was used in another, in conjunction with poly medication therapy. Overall, no recommendations for specific treatments for aggression in HD can be made based on the currently available literature. However, the information contained in this review can be used as a starting reference point for treating clinicians to help determine which medication and non-medication treatments may be suitable for their clients. It can also be used to direct future research with stronger methodological properties.

\section{CONCLUSION}

Aggression is a commonly occurring behavioural sequelae in HD. Further research into the antecedents and precursor "triggers" for this behaviour in HD is required. Given the rarity of the disorder, it is not surprising that treatment studies seeking to reduce aggression in individuals with HD contain small sample sizes. A large randomised controlled trial evaluating the efficacy of treatment for aggression in HD may be difficult to conduct. However, case studies, case series and smaller group studies should be conducted utilising scientifically rigorous methodology. This should include the use of multiple baseline measures of pre-treatment behaviour, quantification of aggressive behaviour using a recognised scale or clearly defined parameters, post treatment evaluation of significance levels and follow-up data to determine the efficacy of the treatment over longer time periods.

\section{CONFLICT OF INTEREST}

The authors do not declare any conflict of interest.

\section{REFERENCES}

[1] Gusella G, Wexler N, Conneally P, Naylor S, Anderson M, Tanzi R, et al. A polymorphic DNA marker genetically linked to Huntington's disease. Nature. 1983;306:234-38.

[2] The Huntington's Disease Collaborative Research Group. A novel gene containing a trinucleotide repeat that is expanded and unstable on Huntington's disease chromosomes. Cell. 1993;72:971-83.

[3] Walker F. Huntington's disease. Lancet. 2007;369(9557):21828.

[4] Brandt J, Butters N. The neuropsychology of Huntington's disease. Trends Neurosci. 1986;9:118-20.

[5] Lemiere J, Decruyenaere M, Evers-Kiebooms G, Vandenbussche E, Dom R. Cognitive changes in patients with Huntington's disease (HD) and asymptomatic carriers of the HD mutation. J Neurol. 2004;251(8):935-42.

[6] Paulsen J, Ready R, Hamilton J, Mega M, Cummings J. Neuropsychiatric aspects of Huntington's disease. J Neurol Neurosurg Psychiatry. 2001;71(3):310-4.

[7] Dewhurst K, Oliver J, McKnight A. Socio-Psychiatric Consequences of Huntington's Disease. Br J Psychiatry. 1970;116(532):255-8

[8] Wheelock V, Tempkin T, Marder K, Nance M, Myers R, Zhao $\mathrm{H}$, et al. Predictors of nursing home placement in Huntington disease. Neurology. 2003;60(6):998-1001.

[9] Rosenblatt A, Leroi I. Neuropsychiatry of Huntington's disease and other basal ganglia disorders. Psychosomatics. 2000;41(1):24-30. 
[10] Cummings J. Behavioral and psychiatric symptoms associated with Huntington's disease. Adv Neurol. 1995;65:179-86

[11] Folstein S, Folstein M. Psychiatric features of Huntington's disease: Recent approaches and findings. Psychiatr Dev. 1983;1(2):193-205.

[12] Bushman B, Anderson C. Methodology in the Study of Aggression: Integrating Experimental and Nonexperimental Findings. In: Geen, RG, Donnerstein E, editor. Human Aggression: Theories, Research, and Implications for Social Policy. San Diego: Academic Press; 1998. pp. 23-48.

[13] Groves M, van Duijn E, Anderson K, Craufurd D, Edmondson MC, Goodman N, et al. An international survey-based algorithm for the pharmacologic treatment of irritability in Huntington's disease. PLoS Curr. 2011;3.

[14] Reedeker N, Bouwens JA, Giltay EJ, Le Mair SE, Roos RAC, van der Mast RC, et al. Irritability in Huntington's disease. Psychiatry Res. 2012;200(2-3):813-8.

[15] Van Duijn E, Craufurd D, Hubers A, Giltay E, Bonelli R, Rickards H, et al. Neuropsychiatric symptoms in a European Huntington's disease cohort (REGISTRY). J Neurol Neurosurg Psychiatry. 2014;85(12):1411-8.

[16] Snaith RP, Taylor CM. Irritability: Definition, assessment and associated factors. Br J Psychiatry. 1985;147(2):127-36.

[17] Holtzman S, O'Connor BP, Barata PC, Stewart DE. The Brief Irritability Test (BITe) A Measure of Irritability for Use Among Men and Women. Assessment. 2014;1073191114533814.

[18] Chatterjee A, Anderson K, Moskowitz C, Hauser W, KS M. A comparison of self-report and caregiver assessment of depression, apathy, and irritability in Huntington's disease. J Neuropsychiatry Clin Neurosci. 2005;17(3): 378-83.

[19] Rosenbaum D. Psychosis with Huntington's chorea. Psychiat Quart. 1941;15:93-9.

[20] Tamir A, Whittier J, Korenyi C. Huntington's chorea: A sex difference in psychopathological symptoms. Dis Nerv Syst. 1969;30(2): 103.

[21] Dewhurst K, Oliver J, Trick K, McKnight A. Neuropsychiatric aspects of Huntington's disease. Confin Neurol. 1969;31(4):258-68

[22] Tyler A, Harper P, Davies K, Newcome R. Family BreakDown and Stress in Huntington's Chorea. J Biosoc Sci. 2008;15(02):127-38.

[23] Burns A, Folstein S, Brandt J, Folstein M. Clinical assessment of irritability, aggression, and apathy in Huntington and Alzheimer disease. J Nerv Ment Dis. 1990;178(1):20-6.

[24] Pflanz S, Beeson J, Ebmeier K, Simpson S. The clinical manifestation of mental disorder in Huntington's disease: A retrospective case record study of disease progression. Acta Psychiatr Scandanavia. 1991;83:53-60.

[25] Shiwach R, Patel V. Aggressive behaviour in Huntington's disease: A cross-sectional study in a nursing home population. Behav Neurol. 1993;6:43-7.

[26] Jensen P, Fenger K, Bolwig T, Sørensen S. Crime in Huntington's disease: A study of registered offences among patients, relatives and controls. J Neurol Neurosurg Psychiatry. 1998;65(4):467-71.

[27] Craufurd D, Thompson J, Snowden J. Behavioral Changes in Huntington Disease. Neuropsychiatry Neuropsychol Behav Neurol. 2001;14(4):219-26.

[28] Grimbergen Y, Knol M, Bloem B, Kremer B, Roos R, Munneke M. Falls and gait disturbances in Huntington's disease. Mov Disord. 2008 May 15;23(7):970-6.

[29] Anderson KE, Gehl CR, Marder KS, Begliner LJ, Paulsen, JS, Huntington's Study Group. Comorbidities of obsessive and compulsive symptoms in Huntington's disease. J Nerv Ment Dis. 2010;198(5):334-8.

[30] Thompson JC, Harris J, Sollom AC, Stopford CL, Howard E, Snowden JS, et al. Longitudinal Evaluation of Neuropsychiatric Symptoms in Huntington's Disease. J Neuropsychiatry Clin Neurosci. 2012;24(1):53-60.

[31] Hubers A, van Duijn E, Roos R, Craufurd D, Rickards H, Bernhard Landwehrmeyer $\mathrm{G}$, et al. Suicidal ideation in a European Huntington's disease population. J Affect Disord. Elsevier; 2013;151(1):248-58

[32] Wetzel H, Gehl C, Dellefave-Castillo L, Schiffman J, Shannon K, Paulsen J. Suicidal ideation in Huntington disease: The role of comorbidity. Psychiatry Res. Elsevier Ltd; 2011;188(3):372-6.

[33] Folstein M, Folstein S, McHugh P. Mini-mental state: A practical method of grading the cognitive state of patients for the clinician. J Psychiatr Res. 1975;12:189-98.

[34] Huntington's Study Group. Unified Huntington's disease rating scale: Reliability and consistency. Mov Disord. 1996;11:136-42.

[35] Patel V, Hope R. A rating scale for assessment of aggression in the elderly-the RAGE. Psychol Med. 1992;22(1): 211-21.

[36] Wing J, Cooper J, Sartorius N. Measurement and classification of psychiatric symptoms. Cambridge: Cambridge Univeristy Press; 1974.

[37] Leonard D, Kidson M, Brown J, Shannon P, Taryan S. A double blind trial of lithium carbonate and haloperidol in Huntington's chorea. Aust NZ J Psychiat. 1975;9(2):115-8.

[38] Stewart J. Adverse behavioral effects of amantadine therapy in Huntington's disease. South Med J. 1987;80(10):1324.

[39] Stewart J. Paradoxical aggressive effect of propranolol in a patient with Huntington's disease. J Clin Psychiatry. 1987;48(3):106-8.

[40] Stewart J, Mounts M, Clark R. Aggressive behavior in Huntington's disease: Treatment with propranolol. J Clin Psychiatry. 1987;48(3):106-8.

[41] Von Hafften A, Jensen C. Paradoxical response to pindolol treatment for aggression in a patient with Huntington's disease. J Clin Psychiatry. 1989;50(6):203-31.

[42] Findling R. Treatment of aggression in juvenile-onset Huntington's disease with buspirone. Psychosomatics. 1993;34(5):460-1.

[43] Byrne A, Martin W, Hnatko G. Beneficial effects of buspirone therapy in Huntington's disease. Am J Psychiatry. 1994;151(7):1994.

[44] Patel S, Tariot P, Asnis J. L-Deprenyl augmentation of fluoxetine in a patient with Huntington's disease. Ann Clin Psychiatry. 1996;8(1):23-6.

[45] Ranen N, Lipsey J, Treisman G, Ross C. Sertraline in the Treatment of Severe Aggressiveness Huntington's Disease in. 1996;(3).

[46] Bhandary A, Masand P. Buspirone in the management of disruptive behaviors due to Huntington's disease and other neurological disorders. Psychosomatics. 1997;38(4):389-91.

[47] Grove V, Quintanilla J, DeVaney G. Improvement of Huntington's disease with olanzapine and valproate. New Engl J Med. 2000;343(13):973-4.

[48] Blass D, Steinberg M, Leroi I, Lyketsos C. Successful Multimodal Treatment of Severe Behavioral Disturbance in a Patient With Advanced Huntington's Disease. Am J Psychiatry. 2001;158(12):1966-72.

[49] Fogliani A, Giorgio A, Di Bonomo V. Awareness of involuntary movements in Huntington disease with olanzapine: A case report. Minerva Psychiatr. 2003;44(3):189-90. 
[50] Alpay M, Koroshetz WJ. Quetiapine in the Treatment of Behavioral Disturbances in Patients With Huntington's Disease. Psychosomatics. 2006;47(1):70-2.

[51] Soliman S, Haque S, George E. Stalking and Huntington's Disease: A Neurobiological Link? J Forensic Sci. 2007;52(5):1202-4.

[52] Edlinger M, Seppi K, Fleischhacker W, Hofer A. Treatment of psychotic and behavioral symptoms with clozapine, aripiprazole, and reboxetine in a patient with Huntington's disease. Int Clin Psychopharmacol. 2013;28(4):214-6.

[53] Rej S, Desautels R. Experience with intramuscular zuclopenthixol and medroxyprogesterone acetate in the treatment of agitation and aggression in Huntington's disease. J Neuropsychiatry Clin Neurosci. 2013;25(3): E33-4.

[54] Ding J, Gadit A. Psychosis with Huntington's disease: Role of antipsychotic medications. BMJ Case Rep. 2014;2014 2013-5.

[55] Brown A, Fisher C. Optimising Occupational Performance through Sensory Modulation: Case reports of two young adults diagnosed with Juvenile Huntington's Disease. Br J Occup Ther. In press. 